\title{
HERBIG-HARO OBJECTS IN THE ORION NEBULA REGION
}

\author{
C. R. O'DELL \\ Max Planck Institute for Astronomy \\ Heidelberg, D-69117 GERMANY
}

\begin{abstract}
The Orion Nebula Region has two different systems of objects classified as $\mathrm{HH}$ objects. The North System is associated with the $\mathrm{H}_{2}$ fingers seen in the infrared and is probably the result of Rayleigh-Taylor instabilities in shocked material moving into the near side of the giant molecular cloud OMC-1. The South System is associated with source(s) within the Trapezium cluster, with the shocked HH objects occuring where jets from pre-main sequence stars impinge on the neutral lid of material that lies across the front of the Orion Nebula. Such jets are different from those driving other $\mathrm{HH}$ objects in that these are passing through photoionized material and two of the Orion jets may have been detected.
\end{abstract}

\section{Introduction}

The Orion Nebula region is fundamentally different from most of the regions where Herbig Haro ( $\mathrm{HH})$ objects are studied. This is because it contains a very high density star cluster, the Trapezium Cluster (Prosser et al. 1994) and this cluster contains many hot, high mass stars. The objects now called HH 203 and HH 204 (Reipurth 1994) are obvious on all well exposed ground images (Münch \& Wilson 1962) and were succeeded in discovery by $\mathrm{HH}$ 201 (Münch \& Taylor 1974), the complex source HH 202 (Cantó et al. 1980), and then numerous sources (HH 205-210) were found in surveys in the [O I] emission line (Axon \& Taylor 1984). A determination of the proper motion of HH 201, 205-6 (Jones \& Walker 1985) indicated that these objects appear to be emanating from a common center. That result was emphasized by the determination by Allen \& Burton (1993) that these last $\mathrm{HH}$ objects are at the tips of radial spokes of molecular hydrogen emission and that there seems to be a common region of geometric origin near the intense infrared source IRc2 (Schild et al. 1997, McCaughrean \& Mac Low 1997). IRc2 seems to be a luminous hot star imbedded within the OMC-1 


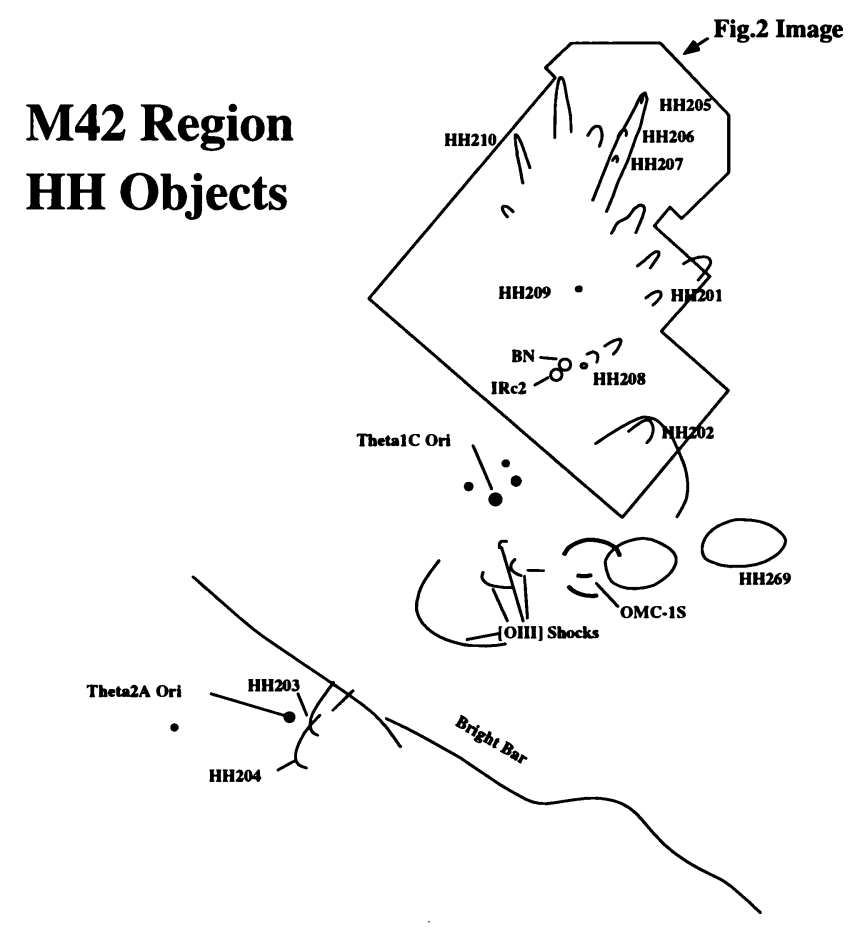

Figure 1. The main features of the Orion Nebula Region are shown, with the brightest stars and infrared and radio sources marked. The angular distance between $\theta^{1} \mathrm{C}$ Ori and $\theta^{2} \mathrm{~A}$ Ori is $135 "$, corresponding to $0.28 \mathrm{pc}$. The cataloged HH objects are numbered and the brightest additional shock forms are also given. The outline shows the region imaged in Figure 2.

molecular cloud. A final entry in the current catalog of $\mathrm{HH}$ objects was added with the determination that $\mathrm{HH} 269$ possesses many of the features of $\mathrm{HH}$ objects, although its morphological form is quite different from all of the others (Walter et al. 1995). Hubble Space Telescope (HST) images of this region (O'Dell \& Wong 1996) have revealed many features that appear to be shocked gas and a special study of the northern part of the nebula has shown that there is a myriad of shock structures. The line drawing shown as Figure 1 illustrates the location of the major features discussed in this paper. 


\section{Structure of the Orion Nebula Region}

An appreciation of the $\mathrm{HH}$ objects we see in the Orion Nebula requires a basic understanding of its three dimensional structure. In the background lies the giant molecular cloud OMC-1, which has imbedded within it the luminous objects producing the IRc2 and Becklin-Neugebauer (BN) infrared sources in the north and the OMC-1S radio source in the south. The Trapezium Cluster lies in the foreground and its hottest member $\theta^{1} \mathrm{C}$ Ori lies at a distance of only $0.2 \mathrm{pc}$ (corresponding to 95 " at the distance of $430 \mathrm{pc}$ ) in front of the cloud surface (O'Dell 1994). The Orion Nebula (M42, NGC $1976)$ is a thin $(0.05 \mathrm{pc})$ zone of ionized material on the front of OMC-1 (Wen \& O'Dell). This "blister" of material is where most of the material of the optical nebula is contained. The recently ionized gas finds itself in an over-pressure situation and freely expands away from the ionization front, so that there is a velocity gradient of about $10 \mathrm{~km} / \mathrm{s}$ as the gas is accelerated away from the ionization front. Although all stages of low through intermediate ionzation are found along the line of sight from $\theta^{1} \mathrm{C}$ Ori to the ionization front, most of the material in the visible nebula is of low ionization and high density, while the material around the young cluster member stars is largely of higher ionization and low density. This is the important difference from other regions of star formation with $\mathrm{HH}$ objects, in that the stars that potentially create the $\mathrm{HH}$ objects exist in an already photoionized gas, which may cause them to appear quite different from most members of this class. In front of the cluster lies a lid of neutral gas and dust, whose presence is revealed by its absorption of 21-cm emission and the formation of absorption lines in the cluster stars (van der Werf \& Goss 1989, O'Dell et al. 1993a). The details of this three dimensional model of the region are summarized in recent articles (Wen \& O'Dell 1995, O'Dell 1994), although the basic model has been progressively refined by many authors since the blister nature of the nebula was first demonstrated over 20 years ago (Zuckerman 1973).

\section{The Two HH Object Systems in this Region}

Study of the various $\mathrm{HH}$ objects here indicate the common property that they all have blueshifted emission lines, although there is a division of the objects in the north and the south, the latter having much lower velocities. In terms of size and form, there also seems to be a division between north and south objects. This leads me to divide the objects into North and South Systems. 


\subsection{THE NORTH SYSTEM}

The North System objects are numerous and relatively homogeneous. The defining feature is their size of a few arcseconds, high blue shifts (several hundred km/s, Axon \& Taylor 1984, Taylor et al. 1986, Hu 1996), and symmetry of placement around the BN-IRc2 location. HH 201, 205-7, and 210 share all these features, and all of them except HH 201 lie at the tip of one of the $\mathrm{H}_{2}$ fingers. Recent HST images made in [O I] and [S II] show (O'Dell et al. 1997) that each of the $\mathrm{H}_{2}$ fingers has associated low ionization optical features and that there are multiple shock form structures in the inner region near IRc2-BN, (Figure 2). HH 208 and HH 209 have the shared features of high blueshifts and small sizes, but do not have the symmetry of a bow shock viewed from an angle. HH 208 has numerous associated shocks out in front of it, and that combined geometry, like HH 201, indicates that the picture is not a simple one of a point of common origin in the IRc2-BN location.

The North System objects probably represent a new subclass of $\mathrm{HH}$ objects, recognizing that most $\mathrm{HH}$ objects known today are shocks driven by jets arising from pre-main sequence stars. The North System complex spans such a wide range of angles but similar dynamic ages that it must be driven by some large scale force. The most attractive mechanism to explain this is that of an intense stellar wind arising in the $\mathrm{O}$ star exciting IRc2, as advocated by Stone (1995). However, in its original formulation, Stone argued that the instabilities that produce the fingers and $\mathrm{HH}$ objects originated from a second fast wind overtaking a previous wind. This would produce fingers and $\mathrm{HH}$ objects in all angles along the line of sight, whereas the fingers all have optical counterparts at their tips, which indicates that they are preferentially located on the near side of OMC-1. The mechanism for explaining this is also in the Stone paper, in that the authors point out that a single stellar wind producing a shock moving into a region of decreasing density would also become Rayleigh-Taylor unstable. Such a density decrease is exactly what one would expect on the near side of OMC1 , so that instabilities in a single wind shock are the most likely origin. The lack of exact orientation with IRc2 is easily accounted for by shaping of the general flow as it passes through inhomogenous regions prior to reaching the observer's side of OMC-1. The North System objects probably should remain in the $\mathrm{HH}$ category, since they still arise from outflow from a young star, and we should be alert that similar objects may be found near other imbedded massive stars. 


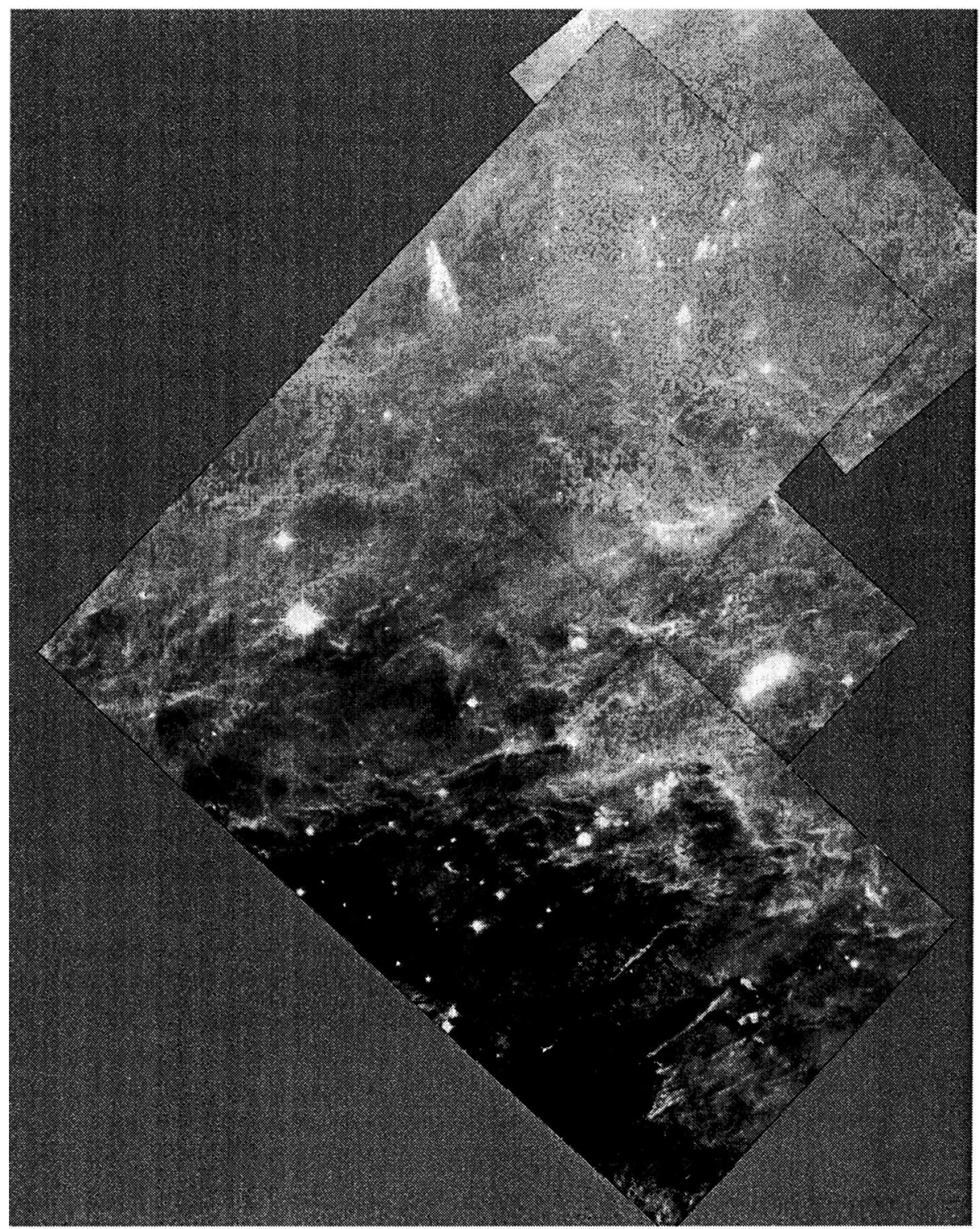

Figure 2. The region recently imaged by HST which includes all of the North System $\mathrm{HH}$ objects plus HH 202. The irregular outline is due to the image being a combination of two different pointings. The image is the sum of $[\mathrm{O} \mathrm{I}]+[\mathrm{S} \mathrm{II}]$ minus $\mathrm{H} \alpha+[\mathrm{N} \mathrm{II}]$, thus subtracting the bright nebular background and enhancing the regions of low excitation. The latter are primarily formed at the shock excited $\mathrm{HH}$ objects and the main ionization front of the nebula. The brightest portions of HH 202 and 205-210 are all saturated in this depiction, in order to display the new faint optical features. 


\subsection{THE SOUTH SYSTEM}

The South System objects include HH 202-4 and HH 269 and their locations are sketched in Figure 1. Again, all of the objects are blueshifted with respect to OMC-1 (O'Dell et al. 1991, O'Dell et al. 1993b, Walter et al. 1995), but are significantly larger and have much lower blueshifts. All probably arise from interactions of jets from one or more pre-main sequence stars with the neutral lid that covers the front of the Orion Nebula, a region that must be much lower density than the OMC-1 surface regions where the North System objects are found. The nature of $\mathrm{HH} 202$ remains uncertain. It has two regions of very high surface brightness placed on a small concave form and is itself a smaller feature enclosed within a much larger concave high ionization structure. HH 203-4 has previously been studied in detail (Hu 1996a,1996b), with HH 203 probably being the result of a jet causing a shock in a cloud with a strong density gradient, i.e. the jet is striking the edge of a cloud. HH 204 is more symmetric and has much fine detail in its tip. All of $\mathrm{HH} \mathrm{202-4}$ have the characteristic of extended [O III] within the concave envelope defining their boundaries. This is very different from what is is expected if only a jet is shocking a neutral cloud. In that case the [O III] emission should be concentrated at the tip, where the least cooling has occured. A natural explanation of this is that the material behind the shock is being photoionized by $\theta^{1} \mathrm{C}$ Ori (O'Dell et al. 1997).

$\mathrm{HH} 269$ (Walter et al. 1995) is different in form from all other $\mathrm{HH}$ objects, being an ellipse oriented almost exactly east-west, and having bright knots at its two tips. The most reasonable explanation is that it is the result of a jet having passed completely through one of the concentrations in the lid, leaving behind only the ring formed well behind the tip of the shock. HST images (O'Dell \& Wen 1994, O'Dell \& Wong 1996 ) indicate that there is a very similar object to its east. That object in turn has to its east a dark incomplete oval of similar form. The bipolar outflow imbedded source OMC-1S lies within the boundaries of this object (Schmid-Burgk et al. 1990, Rodriguez-Franco et al. 1992, Bachiller 1996). It is completely uncertain if this dark object is related to HH 269 and the object in between. A possible clue to the driving source of HH 269 may lie with recent low resolution Fabry-Perot observations (Hartigan et al. 1997) that show there to be a linear "jet" of emission exactly in alignment with the centers of $\mathrm{HH}$ 269 and its eastward companion. On the eastward side of this jet feature are numerous high ionization bow shocks (O'Dell et al. 1993b) whose center of symmetry falls near the west end of the new jet that we see. The symmetry axes of HH 202, 203-4, and 269 intersect in this same region, indicating that this region contains one or more sources of jets. 


\section{Discussion}

The one jet that we do see (driving HH 269) has high ionization. There is a second possible jet extending to the northwest from $\mathrm{HH} 203$ which is similarly ionized. If these are the driving jets, then they are very dissimilar from those previously found. This is not surprising, since any jets would be passing through material that is already ionized. The detection of similar objects in Orion is probably rendered difficult by the strong nebular background. The remaining major question is "Why don't we see HH objects in the South System formed by the blueward jets?" Such objects would arise where those jets impinge on the concentration of material near the ionization front. Because of the high density there the cooling lengths would be short and the objects correspondingly small. The density increase as the jet encounters first the free flow away from the main ionization front and then the photodissociation region means that the mass loading would be very high. Since momentum would be conserved, the corresponding velocity of the shocked material would be little different from its original value, making the shocked material's detection against the nebula very difficult.

In conclusion we can say that the Orion Nebula region is very rich in $\mathrm{HH}$ objects. However, in neither the North or South Systems are we dealing with ordinary HH objects. The North System objects are probably the result of instabilities in a wind-driven shock reaching the decreasing density gradient on the near surface of OMC-1, while the South System objects are the results of jets having passed through photoionized material and finally producing low ionization shocks when they strike the foreground lid that covers the Orion Nebula.

Acknowledgements: This work was supported in part by NASA grant NAG 5-1626. The author was supported in part by the Alexander von Humboldt Foundation while at the Max Planck Institute for Astronomy while on sabbatical leave from Rice University in Houston, Texas. The observations used in this discussion were primarily obtained under STScI program 5976 and the full results of that program are published under the names of all of the team members (O'Dell et al. 1997). Particular thanks are due to team members Patrick Hartigan and Michael G. Burton.

\section{References}

Allen, D. A. \& Burton, M. G. 1993, Nature, 363, 54

Axon, D. J. \& Taylor, K 1984, MNRAS, 207, 241

Bachiller, R. 1996, ARAA, 34111

Cantó, J., Goudis, C., Johnson, P. G., \& Meaburn, J. 1980, A\&A, 85, 128

Hartigan, P., Morse, J. A., \& O'Dell, C. R. 1997, private communication

$\mathrm{Hu}, \mathrm{X} .1996 \mathrm{a}, \mathrm{PhD}$ Thesis, Rice University, Houston, Texas

$\mathrm{Hu}, \mathrm{X} .1996 \mathrm{~b}, \mathrm{AJ}, 112,2712$

Jones, B. R. \& Walker, M. F. 1985, AJ, 90, 1320 
McCaughrean, M. J. \& Mac Low, Mordecai-Mark 1997, AJ, 113, 391

Münch, G. \& Taylor, K. 1974, ApJ, 192, L93

Münch, Guido \& Wilson, O. C. 1962, ZAp, 56, 127

O'Dell, C. R. 1994, ApSS, 216, 267

O'Dell, C. R. \& Wen, Z. 1994, ApJ, 436, 194

O'Dell, C. R. \& Wong, S. K. 1996, AJ, 111, 846

O'Dell, C. R., Wen, Z., \& Hester, J. J. 1991, PASP, 103, 82

O'Dell, C. R., Walter, D. K., \& Dufour, R. J. 1992, ApJ, 399, L67

O'Dell, C. R., Valk, J. H., Wen, Z., \& Meyer, D. M. 1993a, ApJ, 403, 678

O'Dell, C. R., Hartigan, P., W. M. Lane, Wong, S. K., Burton, M. G., Raymond, J., \& Axon, D. J., 1997, AJ, submitted

Prosser, C. F., Stauffer, J. R., Hartmann, L., Soderblom, D. R., Jones, B. F., Werner, M. W., \& McCaughrean, M. J. 1994, ApJ, 421, 517

Reipurth, B. 1994, A General Catalogue of Herbig-Haro Objects, available by electronic transfer through $\mathrm{ftp}$ to hq.eso.org in the directory /pub/Catalogs/Herbig-Haro

Rodriguez-Franco, A., Martin-Pintado, J., Gomez-González, J., \& Planesas, P. 1992, A\&A, 264, 592

Schild, H., Miller, S., \& Tennyson, J. 1997, A\&A, 318, 608

Schmid-Burgk, J., Güsten, R., Mauersberger, R., Schulz, A., \& Wilson, T. L. 1990, ApJ, $362, \mathrm{~L} 25$

Stone, J. M., Xu. J., \& Mundy. L. G. 1995, Nature, 377, 315

van der Werf, P. P. \& Goss, W. M. 1989, A\&A, 224, 209

Walter, D. K., O'Dell, C. R., Hu, X., \& Dufour, R. J. 1995, PASP, 107, 686

Wen, Zheng \& O'Dell, C. R. 1995, ApJ, 438, 784

Zuckerman, B. 1973 ApJ, 183, 863 